\title{
Neoadjuvant Chemotherapy with Gemcitabine Plus Nab-paclitaxel Reduces the Number of Cancer-associated Fibroblasts Through Depletion of Pancreatic Stroma
}

\author{
TOMOHARU MIYASHITA ${ }^{1}$, HIDEHIRO TAJIMA ${ }^{1}$, ISAMU MAKINO ${ }^{1}$, MITSUYOSHI OKAZAKI $^{1}$, \\ TAKAHISA YAMAGUCHI ${ }^{1}$, YOSHINAO OHBATAKE ${ }^{1}$, SINICHI NAKANUMA $^{1}$, \\ HIRONORI HAYASHI ${ }^{1}$, HIROYUKI TAKAMURA ${ }^{1}$, ITASU NINOMIYA ${ }^{1}$, \\ SACHIO FUSHIDA $^{1}$, KOJI KISHIMOTO ${ }^{2}$, JOHN W. HARMON ${ }^{3}$ and TETSUO OHTA ${ }^{1}$ \\ ${ }^{1}$ Department of Gastroenterological Surgery, Kanazawa University Hospital, Kanazawa, Japan; \\ ${ }^{2}$ Graduate School of Technology, Industrial and Social Sciences, Bioscience and Bioindustry, \\ Food Science and Technology, Tokushima University, Tokushima, Japan; \\ ${ }^{3}$ Department of Surgery, Johns Hopkins University School of Medicine, Baltimore, MD, U.S.A.
}

\begin{abstract}
Background: In this study, the effects of neoadjuvant chemotherapy (NAC) on cancer-associated fibroblasts (CAFs) in pancreatic cancer stroma were investigated. Materials and Methods: Density of $\alpha$-smooth muscle actin ( $\alpha S M A)$-positive fibroblasts in resected surgical specimens from untreated patients, patients receiving conventional gemcitabine plus S-1 (GS), and patients receiving gemcitabine plus nab-paclitaxel $(G n P)$ was determined by hybrid cell counting. 18FFluorodeoxyglucose positron-emission tomography (FDG-PET) scans and carbohydrate antigen 19-9 (CA19-9) concentrations were used to assess tumor activity before and after chemotherapy in the GnP group. Results: In this retrospective study of 65 patients, $\alpha S M A$ expression was reduced in the GnP group, as revealed by markedly disorganized collagen and a low density of aSMA-positive fibroblasts. There were significantly fewer $\alpha$ SMA-positive fibroblasts in the GnP than in the untreated and GS groups, but there was no significant difference between the latter two groups. QSMA density reflected a decrease in standardized uptake value on FDG-PET, but not CA19-9 concentration, after GnP chemotherapy. Conclusion: These data suggest that the GnP regimen induces stromal depletion, resulting in fewer CAFs.
\end{abstract}

This article is freely accessible online.

Correspondence to: Tomoharu Miyashita MD, Ph.D., Department of Gastroenterological Surgery, Kanazawa University Hospital, 13-1 Takaramachi, Kanazawa, Ishikawa 920-8641, Japan. Tel: +81 762652362, Fax: +81 762344260. e-mail: tomoharumiya@gmail.com

Key Words: Pancreatic cancer, stroma, chemoresistance, cancerassociated fibroblast, tumor microenvironment.
Pancreatic ductal adenocarcinoma (PDAC), one of the most lethal types of human cancer, is the fourth most common cause of cancer-related deaths in the USA, associated with a 5 -year survival rate of less than 5\% (1). A major factor contributing to this poor prognosis is marked chemoresistance $(2,3)$, which is attributable to multiple factors. Of these factors, desmoplasia and the tumor microenvironment are increasingly being seen as major contributors to chemoresistance of PDAC (4). PDAC is characterized by an abundant stromal response, also known as a desmoplastic reaction, in which the stroma can comprise more than $50 \%$ of the tumor tissue (5). This stroma, aptly termed the tumor microenvironment, characteristically comprises most of the tumor mass and consists of cancerassociated fibroblasts (CAFs), immune cells, small blood vessels, and extracellular matrix (ECM) $(6,7)$. The cellular component of the desmoplastic stroma in PDAC is composed primarily of myofibroblasts, which are characterized by aSMA expression (7). Desmoplasia in tumor tissues promotes tumor development and inhibits drug penetration and uptake (8). The activated stroma index is a novel independent prognostic marker in patients with PDAC undergoing surgery (9). Recent reports have suggested that targeting components of the desmoplastic reaction may enhance the activity of therapeutics targeting tumor cells (10, 11). CAFs are the main effector cells in the desmoplastic reaction, pancreatic stellate cells being the most important source of CAFs (12). The lack of effective therapeutic options and the consequent high mortality rate of PDAC indicate the need to explore other treatment options, such as those aimed at reducing desmoplasia.

Gemcitabine plus nab-paclitaxel $(\mathrm{GnP})$ has been shown to be superior to gemcitabine alone in terms of response, 
progression-free survival, and overall survival in patients with metastatic pancreatic cancer $(13,14)$. GnP reportedly reduces the number of CAFs, inducing marked alterations in cancer stroma, which in turn result in loss of tumor architecture (15).

We compared numbers and characteristics of CAFs and tumor stroma in three groups of patients with PDAC, namely, patients who had received (i) a combination of GnP, (ii) conventional gemcitabine plus oral S-1 (GS), or (iii) no neoadjuvant chemotherapy (NAC).

\section{Materials and Methods}

Patients and tissue samples. Between January 2009 and December 2016, 65 patients with PDAC that had been diagnosed radiologically underwent surgery at the Department of Gastroenterological Surgery of Kanazawa University Hospital. Thirty-six of them had pancreatic head cancer and 29 with cancer of the pancreatic body and tail. They comprised 43 men and 22 women of average age 69 years (range $=48-84$ years). Twenty-three out of the 65 had no chemotherapy, 23 had GS, and 19 had GnP before undergoing surgical resection (Table I).

The GnP group received $75 \mathrm{mg} / \mathrm{m}^{2}$ nab-paclitaxel followed by $1000 \mathrm{mg} / \mathrm{m}^{2}$ gemcitabine on days 1,8 , and 15 for two 28 -day cycles. ${ }^{18}$ F-Fluorodeoxyglucose positron-emission tomography (FDG-PET) scan and carbohydrate antigen 19-9 (CA19-9) were used to determine tumor status before and after two cycles of treatment. The GS regime comprised $50 \mathrm{mg} / \mathrm{m}^{2} \mathrm{TS}-1$ on days $1-14$ and $1000 \mathrm{mg} / \mathrm{m}^{2}$ gemcitabine on days 8 and 15 for two 21-day cycles, as previously reported (16). Patients with stable or responding tumors were scheduled for surgical resection 6-8 weeks after the last chemotherapy dose. A complete medical history, physical examination, complete blood counts, serum chemistry, serum CA19-9 concentration and high-resolution computed tomographic (CT) scan of the abdomen were obtained for all patients. FDG-PET scans were obtained only for patients in the $\mathrm{GnP}$ group.

Ethics statement. The study was approved by the Ethics Committee of the Kanazawa University Hospital (UMIN000011062). Written informed consent was obtained from each patient enrolled in the study.

Pathological specimens. Paraffin-embedded operative tissue samples of the 65 patients with PDAC were obtained from the Institute of Pathology for further immunohistochemical analysis. The specimens had previously been fixed in $10 \%$ formalin and embedded in paraffin. Several 3- $\mu$ m-thick sections were cut from each paraffin block; one was stained with hematoxylin and eosin and the other was subjected to immunohistochemical staining for $\alpha$-smooth muscle actin ( $\alpha$-SMA).

Immunohistochemical examination. The Dako Envision system (Dako, Carpinteria, CA, USA), which uses dextran polymers conjugated to horseradish peroxidase, was employed for immunohistochemical staining to avoid any endogenous biotin contamination. Sections were deparaffinized in xylene and rehydrated in a graded ethanol series. Endogenous peroxidase was blocked by immersing sections in $3 \% \mathrm{H}_{2} \mathrm{O}_{2}$ in $100 \%$ methanol for
Table I. Patient characteristics according to therapy group.

\begin{tabular}{|c|c|c|c|c|}
\hline & $\begin{array}{l}\text { Untreated } \\
\qquad(\mathrm{n}=23)\end{array}$ & $\begin{array}{c}\text { GS } \\
(\mathrm{n}=23)\end{array}$ & $\underset{(\mathrm{n}=19)}{\mathrm{GnP}}$ & $p$-Value \\
\hline Median age (range), years & $69(52-84)$ & 66 (54-78) & $69(48-80)$ & 0.124 \\
\hline Gender & & & & 0.31 \\
\hline Male & 18 & 14 & 11 & \\
\hline Female & 5 & 9 & 8 & \\
\hline Location & & & & 0.54 \\
\hline Pancreatic head & 12 & 15 & 9 & \\
\hline Pancreatic body/tail & 11 & 8 & 10 & \\
\hline Differentiation & & & & 0.98 \\
\hline Well/moderate & 19 & 20 & 16 & \\
\hline Poor & 2 & 2 & 2 & \\
\hline Other & 2 & 1 & 1 & \\
\hline UICC stage & & & & 0.68 \\
\hline I & 1 & 2 & 1 & \\
\hline II & 21 & 19 & 18 & \\
\hline III & 1 & 2 & 0 & \\
\hline Resectability & & & & 0.92 \\
\hline 0 & 18 & 19 & 16 & \\
\hline $1<$ & 5 & 4 & 3 & \\
\hline
\end{tabular}

GS, Gemcitabine plus S-1; GnP, gemcitabine plus nab-paclitaxel; UICC, Union for International Cancer Control.

$20 \mathrm{~min}$ at room temperature. Antigen retrieval was achieved by microwaving sections at $95^{\circ} \mathrm{C}$ for $10 \mathrm{~min}$ in $0.001 \mathrm{M}$ citrate buffer (pH 6.7). After blocking endogenous peroxidase, sections were incubated with Protein Block Serum-Free (Dako) at room temperature for $10 \mathrm{~min}$ to block non-specific staining. Subsequently, sections were incubated for $2 \mathrm{~h}$ at room temperature with 1:100 diluted monoclonal mouse antibody against human $\alpha$ SMA (Clone 1A4; Dako). Peroxidase activity was detected using the enzyme substrate 3-amino-9-ethylcarbazole. Sections were incubated in Trisbuffered saline without primary antibodies for negative controls. Samples were faintly counterstained with Meyer's hematoxylin.

Evaluation of immunostaining. A fibroblast density index was determined by relating the observed proportion of tumor fibrosis to the number of cells counted in each specimen. The amount of staining in the stroma was assessed using Hybrid Cell Count BZH2C software (Keyence, Osaka, Japan) according to the developer's protocol. Digital images were analyzed for the positively stained area versus the total surface area using the hybrid cell count function. Color artifacts and major arteries were excluded manually from the analysis by the investigator. Quantification was performed in five different tumor regions and representative high-power fields from each sample. Detected cells were classified according to the ratio of mean layer intensities of $\alpha \mathrm{SMA}$ as described by Erkan $e t$ al. (9). Results are expressed as total stained area/total surface area.

Statistical analysis. Differences were analyzed for significance using the chi-square test, Mann-Whitney $U$-test, or log-rank test, as appropriate. Data management and statistical analysis were performed using SPSS version 15 software (SPSS, Chicago, IL, USA). $p$-Values of less than 0.05 were considered to denote statistical significance. 


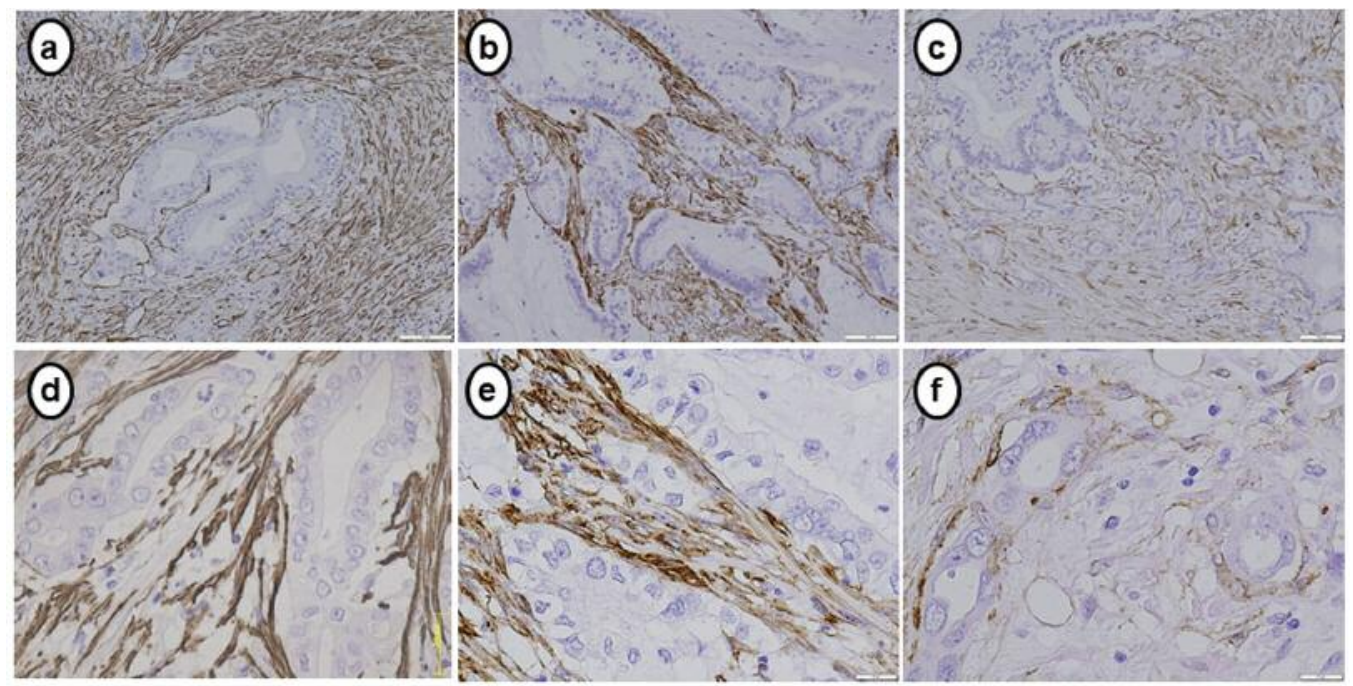

Figure 1. $\alpha$-Smooth muscle actin ( $\alpha S M A)$ expression according to treatment group. Representative photomicrographs of sections stained immunohistologically for $\alpha S M A-p o s i t i v e$ fibroblasts in the neighborhood of cancer glands (brown). $\alpha$ SMA expression in untreated patients ( $a, \times 20$; $d, \times 40)$ and in those treated with gemcitabine plus $S-1(b, \times 20 ; e, \times 40)$ is reflected by long, continuous, well-organized, and overlapping cancerassociated fibroblasts $(C A F s)$, whereas in the group treated with gemcitabine plus nab-paclitaxel $(c, \times 20 ; f, \times 40)$, CAFs were less abundant.

\section{Results}

Tumor tissue characteristics in GnP-treated, GS-treated and untreated control groups. $\alpha$ SMA expression in tumor tissue samples from patients who had received no neoadjuvant chemotherapy, GS, or GnP was analyzed. In the GnP group, there was little $\alpha$ SMA-positive fibrosis (i.e. $\alpha \mathrm{SMA}$ expression) related to CAFs around tumor glands. In contrast, strong aSMA expression was apparent in tumor tissue from untreated patients and the group treated with GS, as evidenced by long, continuous, well-organized, and overlapping CAFs (Figure 1).

Patients who had been treated with GnP had a lower density of $\alpha$ SMA-positive cells. The average $\alpha$ SMA density in the group treated with $\mathrm{GnP}$ was $16 \% \pm 8 \%$, whereas it was $40 \% \pm 8 \%$ and $38 \% \pm 13 \%$, respectively, in samples from untreated patients and patients treated with GS. $\alpha$ SMA density was significantly lower in the group treated with $\mathrm{GnP}$ than in the untreated or GS-treated groups (both $p<0.0001)$. However, $\alpha$ SMA density did not differ significantly between the untreated and GS groups $(p=0.148)$ (Figure 2).

Relationship between NAC and survival outcomes. Overall survival in the different groups are shown in Figure 3. Overall survival did not differ significantly between the untreated group and that treated with GS group $(p=0.81)$ or GnP group $(p=0.72)$, nor between those treated with GS and $\mathrm{GnP}(p=0.92)$ (Figure 3).
Relationship between FDG-PET, CA19-9 evidence of clinical activity, and GnP in patients with PDAC. Relevant characteristics of the 19 patients who received the specified two cycles of GnP before surgical resection are presented in Table I. Six out of these 19 patients had $\alpha$ SMA density $>20 \%$ and in the other 13 it was $<20 \%$. Three of the latter had negative FDG-PET findings before chemotherapy, whereas four of the remaining 16 patients had $\alpha$ SMA density $>20 \%$ and the other $12<20 \%$. All patients with increased maximum standardized uptake values (SUVs) on FDG after chemotherapy had $\alpha$ SMA density $>20 \%$. In contrast, only two out of 14 patients with decreased maximum SUV on FDG after treatment had $\alpha$ SMA density $>20 \%$ (Figure 4a-c). Thus, $\alpha$ SMA density reflected the degree of decrease in SUV on FDG-PET $(p=0.008)$ (Figure 4c). Regarding CA19-9 concentrations, two out of 19 patients were negative for CA19-9 before chemotherapy. One patient with increased CA19-9 concentration after chemotherapy had aSMA density $>20 \%$, whereas five out of 16 patients with decreased CA19-9 concentration after chemotherapy had $\alpha$ SMA density $>20 \%$. No relationship between CA19-9 concentration and $\alpha$ SMA density was identified $(p=0.16)$ (Figure 4d).

\section{Discussion}

The findings of this study indicate that therapy with GnP induces a much more pronounced decrease in CAF content in PDAC than occurs in untreated patients or those treated 


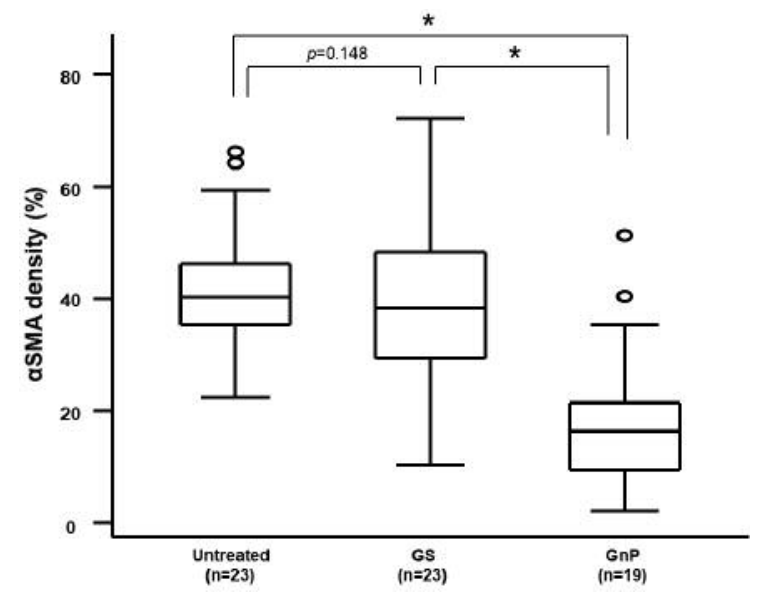

Figure 2. $\alpha$-Smooth muscle actin ( $\alpha S M A)$ density according to treatment group. Median aSMA-positive density index was determined by dividing the proportion of $\alpha S M A$-positive fibroblasts by stromal content in five random high-power fields. As shown, $\alpha$ SMA density was significantly lower in the activated group treated with gemcitabine plus nabpaclitaxel (GnP) than in the untreated and gemcitabine plus $S-1(G S)$ treated groups. Mann-Whitney U-test was used for statistical analysis: *significantly different at $p<0.0001$.

with conventional GS. Furthermore, the stromal response was reflected by FDG-PET findings. However, overall survival did not differ between the three treatment groups.

In PDAC, strong myofibroblastic stromal reactions resulting in a so-called 'desmoplastic stroma' are usually associated with higher-grade malignancies and poor outcomes (17). CAFs, which have an 'activated fibroblast phenotype', are cellular components in most of the desmoplastic stroma. There is some evidence that CAFs are characterized by a distinct gene-expression profile and that their properties differ from those of normal fibroblasts (18). PDAC is characterized by development of a desmoplastic reaction, features of which include CAFs, ECM components, and immune and endothelial cells. In PDAC tumors, interactions between cancer and stromal cells reportedly contribute to enhancing growth, invasion, metastasis, and chemoresistance (19). The tumor microenvironment or desmoplastic stroma in PDAC is home to many cellular and molecular components. Reciprocal growth factor signaling results in strong expression of growth factors such as transforming growth factor (TGF)- $\beta$, platelet-derived growth factor (PDGF), and epidermal growth factor (EGF) (20). Impaired paracrine signaling between CAFs and cancer cells and alterations in the ECM microenvironment caused by depletion of CAFs may directly contribute to changes in cancer cells, leading to acquisition of epithelialmesenchymal transition (EMT), a stem cell-like phenotype, and dedifferentiation. In a previous study, we found extravasated platelet aggregation (EPA) in the stroma at the

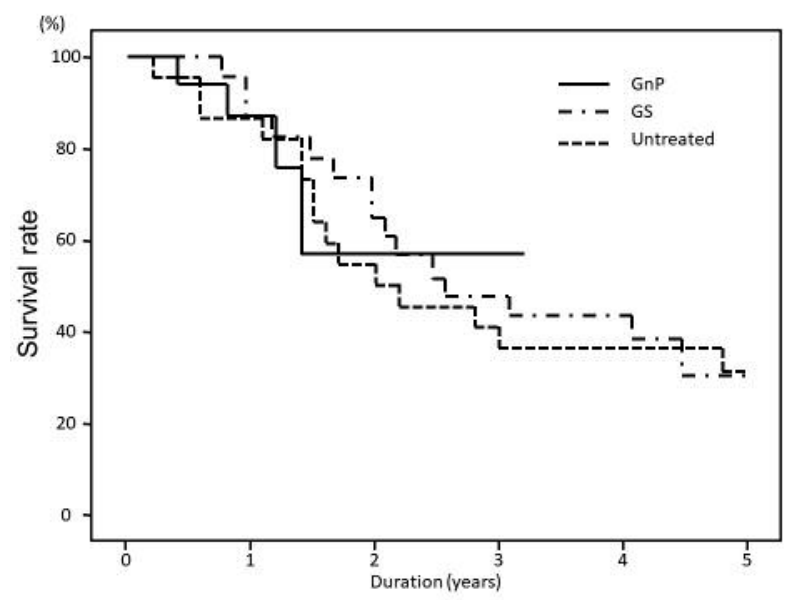

Figure 3. Overall survival according to treatment group. There were no significant differences in overall survival between the three group: log-rank test: Untreated vs. gemcitabine plus $S-1$ (GS), $p=0.81$; untreated vs. gemcitabine plus nab-paclitaxel (GnP), $p=0.72 ; G S$ vs. $G n P, p=0.92$.

invasive fronts of PDAC tumors. Because platelets contain storage pools of growth factors such as PDGF, TGF- $\beta$, VEGF, thrombospondin-1, and plasminogen activator inhibitor-1, EPA is a component of the first step in the process of EMT. Accordingly, we reported that EPA may provide important insights into new therapeutic targets in PDAC stroma (21). Thus, targeting desmoplastic stroma, which is a response of the tumor microenvironment to molecular cues from the cellular compartment, could be a viable component of therapeutic strategies against PADC (8).

In our previous study, low-dose paclitaxel ameliorated stromal fibrosis of human peritoneal mesothelial cells via suppressing the TGF- $\beta$ /contraction of Sma and Mad (SMAD) signaling pathway by inhibiting SMAD2 phosphorylation (22). High-pressure homogenization of paclitaxel with nanoparticle albumin results in formation of soluble $130 \mathrm{~nm}$ complexes in which albumin acts as a carrier for the otherwise hydrophobic paclitaxel. It has been hypothesized that nab-paclitaxel utilizes endogenous transport pathways for albumin to achieve enhanced drug delivery and tumor tissue distribution. Once these complexes reach the tumor milieu, they deplete the tumor stroma. Additionally, paclitaxel is transported into tumor cells along with albumin, where it then exerts its antineoplastic activity (23). However, the effects of nab-paclitaxel in PDAC stroma are not fully understood and, to some extent, controversial. Here we showed by examining samples of PDAC from patients treated with GnP that the predominant effect of this regimen is stromal remodeling resulting in marked alteration of stromal architecture and 

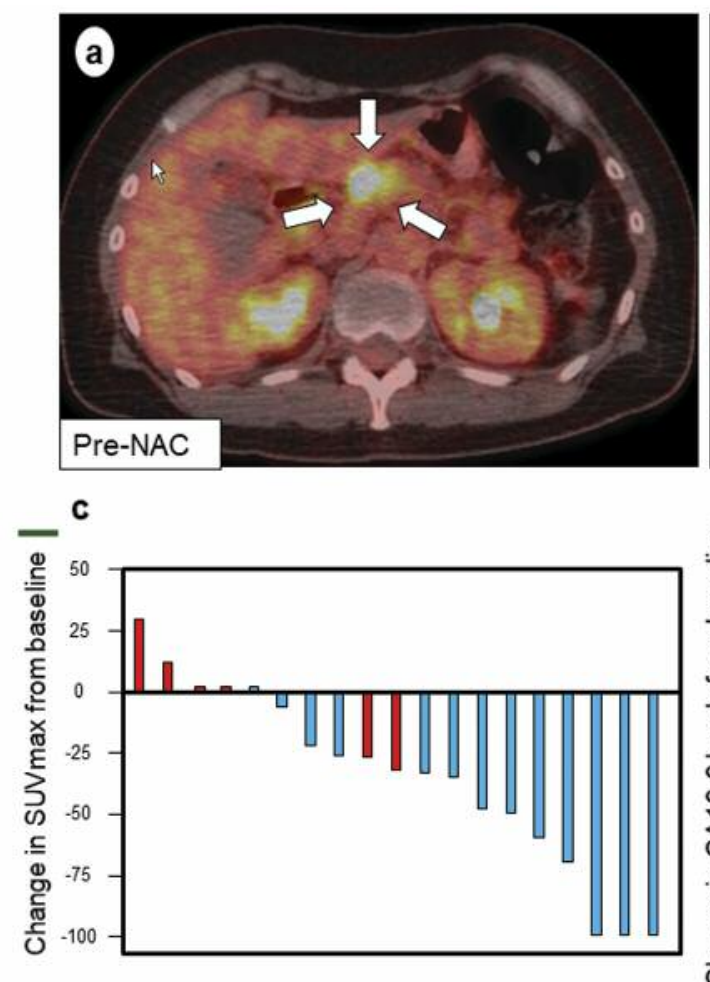

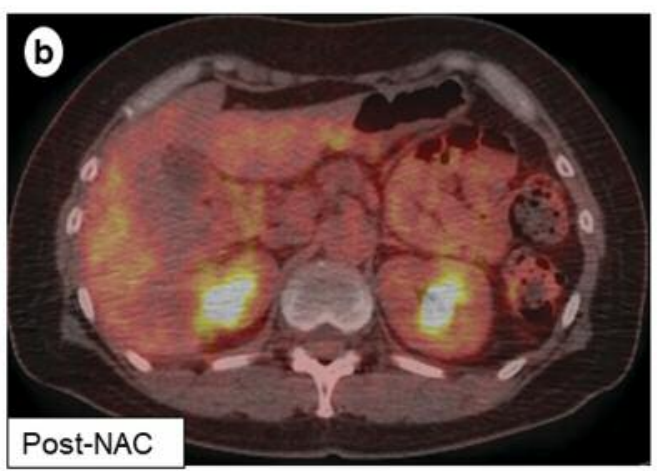

d

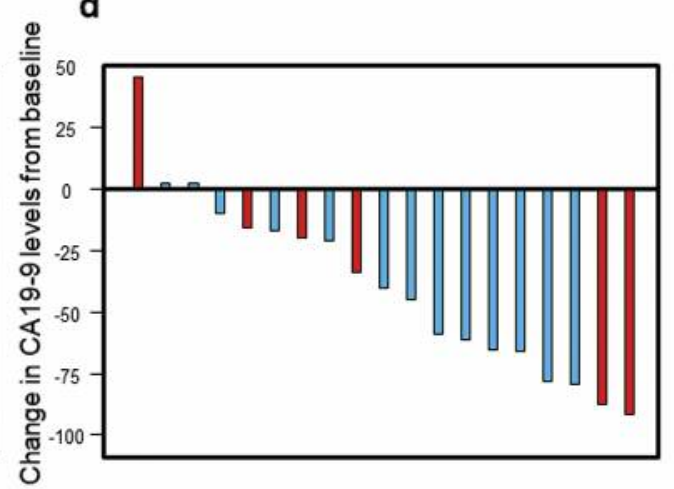

Figure 4. Relationship between clinical activity and gemcitabine plus nab-paclitaxel (GnP) in pancreatic ductal adenocarcinoma. Representative $18 F$-fluorodeoxyglucose (FDG) positron-emission tomography/computed tomographic scan images. The primary tumor (white arrows) before treatment $(a)$, and after treatment $(b)$. Panels $(c)$ and $(d)$ show waterfall plots of changes from baseline in standardized uptake value (SUV) FDG uptake and carbohydrate antigen 19-9 (CA19-9) tumor marker ratio of patients treated with GnP. Studies were performed at baseline, before treatment, and after two cycles of neoadjuvant chemotherapy (NAC); best responses are shown. Red: $\alpha$-Smooth muscle actin ( $\alpha$ SMA) density greater than 20\%; blue, aSMA density less than $20 \%$.

elimination of CAFs. This is best reflected by changes in CAF density, which appears to differ in untreated patients and those treated with conventional GS. The nab-paclitaxel also increases the amount of gemcitabine inside tumor cells by inhibiting cytidine deaminase, the enzyme that degrades gemcitabine (24). Because gemcitabine efficacy is reportedly unaltered in myofibroblast and type I collagen-depleted tumors (25), nab-paclitaxel is capable of altering the stroma in PDAC. In a previous study, we found that low-dose gemcitabine induces expression of major histocompatibility complex class I-related chain $\mathrm{A} / \mathrm{B}$, thus enhancing innate immune function in patients with PDAC (26). In Japan, gemcitabine, and S-1 are now regarded as the key drugs for managing PDAC (27). A large-scale phase III study (GEST study) conducted in patients with metastatic or locally advanced pancreatic cancer in Japan and Taiwan found that GS therapy did not achieve better overall survival than gemcitabine alone (28). However, the MPACT trial, a phase III trial, showed that GnP is superior to gemcitabine alone in terms of response, progression-free survival, and overall survival in patients with metastatic pancreatic cancer (13).
Surgical resection offers the only chance of cure for nonmetastatic pancreatic cancer. NAC prior to resection is associated with a significant survival benefit compared with upfront resection in patients with early-stage, resected PDAC (29). Several randomized studies comparing various NAC regimens with upfront surgery are in progress around the world. A phase III trial (Prep-02/JSAP05) comparing NAC with GS followed by surgery versus upfront surgery in patients with resectable PDAC is expected to provide definitive results in terms of survival. The NEONAX study (AIO-PAK-0313), a phase II study in Germany, started recruiting patients with resectable pancreatic cancer in April 2015. This trial will randomize 166 patients to either perioperative treatment with nab-paclitaxel and gemcitabine ( 2 months before surgery and 4 months after surgery) or adjuvant treatment with $\mathrm{GnP}(30)$. It is important to wait for the outcomes of these trials. In this study, the GnP regimen induced markedly fewer CAFs in PDAC than in untreated patients or those treated with conventional GS. However, overall survival did not differ between the three treatment groups. This study had several limitations, 
including its retrospective nature, small sample size, potential selection bias for surgery, and heterogeneity of tumor characteristics.

FDG-PET/CT may have a role in staging and survival prediction of PDAC and may add to other diagnostic information, such as that provided by histology (31). Low FDG uptake in PET scans of patients with stage I and II pancreatic cancer correlates with superior overall survival and recurrence-free survival, suggesting that glucose metabolic pathways are important in pancreatic cancer biology and that PET scan activity can be used as a prognostic biomarker after pancreatectomy (32). CA19-9 is the only validated tumor marker in widespread clinical use; however, there are few precise data concerning its role in pancreatic cancer diagnosis, staging, determining resectability, response to chemotherapy and prognosis (33). In this study, FDG-PET findings reflected lower aSMA density,but not CA19-9 concentration.

In conclusion, the treatment regimen of GnP may induce stromal depletion, resulting in decreased CAF presence in patients with PDAC. The present findings suggest a potential role for $\mathrm{GnP}$ in suppressing chemoresistance and metastasis by altering the tumor microenvironment. We are now conducting research to elucidate the mechanisms of chemoresistance with the aim of discovering and validating novel therapeutic targets.

\section{Conflicts of Interest}

The Authors declare they have no financial or other conflicts of interest in relation to the content of this article.

\section{Acknowledgements}

The present study was supported by the Japan Society for the Promotion of Science (KAKENHI grant no. 17K10693).

\section{References}

1 Jemal A, Siegel R, Xu J and Ward E: Cancer statistics, 2010. CA Cancer J Clin 60(5): 277-300, 2010.

2 Zalatnai A and Molnár J: Review. Molecular background of chemoresistance in pancreatic cancer. In Vivo 21(2): 339-347, 2007.

3 McCarroll JA, Naim S, Sharbeen G, Russia N, Lee J, Kavallaris M, Goldstein D and Phillips PA: Role of pancreatic stellate cells in chemoresistance in pancreatic cancer. Front Physiol 5: 141, 2014.

4 Schober M, Jesenofsky R, Faissner R, Weidenauer C, Hagmann W, Michl P, Heuchel RL, Haas SL and Löhr JM: Desmoplasia and chemoresistance in pancreatic cancer. Cancers 6(4): 21372154, 2014.

5 Apte MV, Park S, Phillips PA, Santucci N, Goldstein D, Kumar RK, Ramm GA, Buchler M, Friess H, McCarroll JA, Keogh G, Merrett N, Pirola R and Wilson JS: Desmoplastic reaction in pancreatic cancer: Role of pancreatic stellate cells. Pancreas 29(3): 179-187, 2004.
6 Kadaba R, Birke H, Wang J, Hooper S, Andl CD, Di Maggio F, Soylu E, Ghallab M, Bor D, Froeling FE, Bhattacharya S, Rustgi AK, Sahai E, Chelala C, Sasieni P and Kocher HM: Imbalance of desmoplastic stromal cell numbers drives aggressive cancer processes. J Pathol 230(1): 107-117, 2013.

7 Feig C, Gopinathan A, Neesse A, Chan DS, Cook N and Tuveson DA: The pancreas cancer microenvironment. Clin Cancer Res 18(16): 4266-4276, 2012.

8 Whatcott CJ, Diep CH, Jiang P, Watanabe A, LoBello J, Sima C, Hostetter G, Shepard HM, Von Hoff DD and Han H: Desmoplasia in primary tumors and metastatic lesions of pancreatic cancer. Clin Cancer Res 21(15): 3561-3568, 2015.

9 Erkan M, Michalski CW, Rieder S, Reiser-Erkan C, Abiatari I, Kolb A, Giese NA, Esposito I, Friess H and Kleeff J: The activated stroma index is a novel and independent prognostic marker in pancreatic ductal adenocarcinoma. Clin Gastroenterol Hepatol 6(10): 1155-1161, 2008.

10 Provenzano PP, Cuevas C, Chang AE, Goel VK, Von Hoff DD and Hingorani SR: Enzymatic targeting of the stroma ablates physical barriers to treatment of pancreatic ductal adenocarcinoma. Cancer Cell 21(3): 418-429, 2012.

11 Von Hoff DD, Ramanathan RK, Borad MJ, Laheru DA, Smith LS, Wood TE, Korn RL, Desai N, Trieu V, Iglesias JL, Zhang H, Soon-Shiong P, Shi T, Rajeshkumar NV, Maitra A and Hidalgo M: Gemcitabine plus nab-paclitaxel is an active regimen in patients with advanced pancreatic cancer: A phase I/II trial. J Clin Oncol 29(34): 4548-4554, 2011.

12 Nielsen MF, Mortensen MB and Detlefsen S: Key players in pancreatic cancer-stroma interaction: Cancer-associated fibroblasts, endothelial and inflammatory cells. World J Gastroenterol 22(9): 2678-2700, 2016.

13 Von Hoff DD, Ervin T, Arena FP, Chiorean EG, Infante J, Moore M, Seay T, Tjulandin SA, Ma WW, Saleh MN, Harris M, Reni M, Dowden S, Laheru D, Bahary N, Ramanathan RK, Tabernero J, Hidalgo M, Goldstein D, Van Cutsem E, Wei X, Iglesias J and Renschler MF: Increased survival in pancreatic cancer with nabpaclitaxel plus gemcitabine. N Engl J Med 369(18): 1691-1703, 2013.

14 Goldstein D, El-Maraghi RH, Hammel P, Heinemann V, Kunzmann V, Sastre J, Scheithauer W, Siena S, Tabernero J, Teixeira L, Tortora G, Van Laethem JL, Young R, Penenberg DN, Lu B, Romano A and Von Hoff DD: Nab-paclitaxel plus gemcitabine for metastatic pancreatic cancer: Long-term survival from a phase III trial. J Natl Cancer Inst 107(2): dju413, 2015.

15 Alvarez R, Musteanu M, Garcia-Garcia E, Lopez-Casas PP, Megias D, Guerra C, Muñoz M, Quijano Y, Cubillo A, Rodriguez-Pascual J, Plaza C, de Vicente E, Prados S, Tabernero S, Barbacid M, Lopez-Rios F and Hidalgo M: Stromal disrupting effects of nab-paclitaxel in pancreatic cancer. $\mathrm{Br} \mathrm{J}$ Cancer 109(4): 926-933, 2013.

16 Uesaka K, Boku N, Fukutomi A, Okamura Y, Konishi M, Matsumoto I, Kaneoka Y, Shimizu Y, Nakamori S, Sakamoto H, Morinaga S, Kainuma O, Imai K, Sata N, Hishinuma S, Ojima H, Yamaguchi R, Hirano S, Sudo T, Ohashi Y and Group JS: Adjuvant chemotherapy of S-1 versus gemcitabine for resected pancreatic cancer: A phase 3, open-label, randomised, noninferiority trial (JASPAC 01). Lancet 388(10041): 248-257, 2016.

17 Shimoda M, Mellody KT and Orimo A: Carcinoma-associated fibroblasts are a rate-limiting determinant for tumour progression. Semin Cell Dev Biol 21(1): 19-25, 2010. 
18 Allinen M, Beroukhim R, Cai L, Brennan C, Lahti-Domenici J, Huang HY, Porter D, Hu M, Chin L, Richardson A, Schnitt S, Sellers WR and Polyak K: Molecular characterization of the tumor microenvironment in breast cancer. Cancer Cell 6(1): 1732, 2004.

19 Whatcott C, Han H, Posner RG and Von Hoff DD: Tumorstromal interactions in pancreatic cancer. Crit Rev Oncog 18(12): 135-151, 2013.

20 Shimizu K: Mechanisms of pancreatic fibrosis and applications to the treatment of chronic pancreatitis. J Gastroenterol 43(11): 823-832, 2008.

21 Miyashita T, Tajima H, Makino I, Nakagawara H, Kitagawa H, Fushida S, Harmon JW and Ohta T: Metastasis-promoting role of extravasated platelet activation in tumor. J Surg Res 193(1): 289-294, 2015.

22 Tsukada T, Fushida S, Harada S, Terai S, Yagi Y, Kinoshita J, Oyama K, Tajima H, Ninomiya I, Fujimura T and Ohta T: Lowdose paclitaxel modulates tumour fibrosis in gastric cancer. Int J Oncol 42(4): 1167-1174, 2013.

23 Hawkins MJ, Soon-Shiong P and Desai N: Protein nanoparticles as drug carriers in clinical medicine. Adv Drug Deliv Rev 60(8): 876-885, 2008.

24 Chen N, Brachmann C, Liu X, Pierce DW, Dey J, Kerwin WS, Li Y, Zhou S, Hou S, Carleton M, Klinghoffer RA, Palmisano $\mathrm{M}$ and Chopra R: Albumin-bound nanoparticle (nab) paclitaxel exhibits enhanced paclitaxel tissue distribution and tumor penetration. Cancer Chemother Pharmacol 76(4): 699-712, 2015

25 Ozdemir BC, Pentcheva-Hoang T, Carstens JL, Zheng XF, Wu CC, Simpson TR, Laklai H, Sugimoto H, Kahlert C, Novitskiy SV, De Jesus-Acosta A, Sharma P, Heidari P, Mahmood U, Chin L, Moses HL, Weaver VM, Maitra A, Allison JP, LeBleu VS and Kalluri R: Depletion of carcinoma-associated fibroblasts and fibrosis induces immunosuppression and accelerates pancreas cancer with reduced survival. Cancer Cell 25(6): 719-734, 2014.

26 Miyashita T, Miki K, Kamigaki T, Makino I, Nakagawara H, Tajima H, Takamura H, Kitagawa H, Fushida S, Ahmed AK, Duncan MD, Harmon JW and Ohta T: Low-dose gemcitabine induces major histocompatibility complex class I-related chain $\mathrm{a} / \mathrm{b}$ expression and enhances an antitumor innate immune response in pancreatic cancer. Clin Exp Med 17(1): 19-31, 2017
27 Sudo K, Nakamura K and Yamaguchi T: S-1 in the treatment of pancreatic cancer. World J Gastroenterol 20(41): 15110-15118, 2014.

28 Ueno H, Ioka T, Ikeda M, Ohkawa S, Yanagimoto H, Boku N, Fukutomi A, Sugimori K, Baba H, Yamao K, Shimamura T, Sho M, Kitano M, Cheng AL, Mizumoto K, Chen JS, Furuse J, Funakoshi A, Hatori T, Yamaguchi T, Egawa S, Sato A, Ohashi Y, Okusaka T and Tanaka M: Randomized phase III study of gemcitabine plus S-1, S-1 alone, or gemcitabine alone in patients with locally advanced and metastatic pancreatic cancer in Japan and Taiwan: GEST study. J Clin Oncol 31(13): 1640-1648, 2013.

29 Mokdad AA, Minter RM, Zhu H, Augustine MM, Porembka MR, Wang SC, Yopp AC, Mansour JC, Choti MA and Polanco PM: Neoadjuvant therapy followed by resection versus upfront resection for resectable pancreatic cancer: A propensity scorematched analysis. J Clin Oncol, 2016, [Epub ahead of print].

30 Ettrich TJ, Perkhofer L and Seufferlein T: Therapy in pancreatic cancer - and still it moves along!. Dtsch Med Wochenschr 140(7): 508-511, 2015 (in German).

31 Rijkers AP, Valkema R, Duivenvoorden HJ and van Eijck $\mathrm{CH}$ : Usefulness of F-18-fluorodeoxyglucose positron-emission tomography to confirm suspected pancreatic cancer: A metaanalysis. Eur J Surg Oncol 40(7): 794-804, 2014.

32 Pimiento JM, Davis-Yadley AH, Kim RD, Chen DT, Eikman EA, Berman CG and Malafa MP: Metabolic activity by $18 \mathrm{~F}-$ FDG-PET/CT is prognostic for stage I and II pancreatic cancer. Clin Nucl Med 41(3): 177-181, 2016.

33 Winter JM, Yeo CJ and Brody JR: Diagnostic, prognostic, and predictive biomarkers in pancreatic cancer. J Surg Oncol 107(1): 15-22, 2013.
Received October 12, 2017

Revised November 3, 2017

Accepted November 7, 2017 